
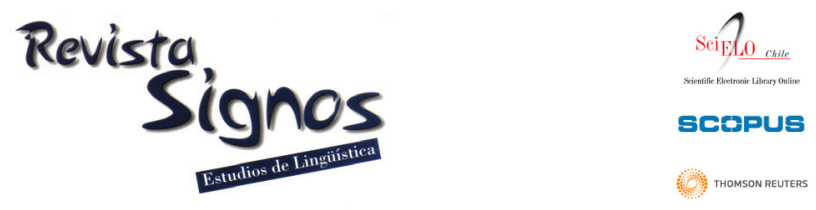

\title{
Control de la Comprensión micro y macro-estructural durante la lectura de textos científicos en lengua extranjera: ¿Algo más que dominio del idioma?
}

\section{Micro and macro-structural comprehension monitoring when reading science texts in a foreign language: Is it just a proficiency matter?}

\author{
Ángela Gómez \\ Universidad de VALENCIA \\ EsPAÑA \\ angela.gomez@uv.es
}

\author{
Vicente Sanjosé \\ Universidad de VALENCIA \\ EsPAÑA \\ vicente.sanjose@uv.es
}

Recibido: 9-IV-2012 / Aceptado: 10-X-2012

\section{Resumen}

Se presenta un estudio con estudiantes universitarios españoles con dominios básico, intermedio y avanzado de inglés como lengua extranjera. El objetivo fue estudiar la efectividad en el Control de la Comprensión (CC) en inglés, comparado con el que se tiene en español, y explicar los resultados a partir del modelo de comprensión de textos desarrollado por Kintsch y otros colegas. Nuestro foco de atención fue la representación semántica, diferenciando el CC a nivel micro y macroestructural. Se midió el CC al leer textos asumiendo el Error Detection Paradigm: se insertaron inconsistencias micro y macroestructurales en los textos y se pidió a los estudiantes juzgar su comprensibilidad usando un código para distinguir entre 'palabras desconocidas', 'ideas de significado absurdo' e 'ideas incoherentes o incompatibles con otras del texto'. Se utilizaron textos de ciencias, tres en inglés y otros tres en español para aumentar la fiabilidad. Las predicciones fueron: a) en inglés, los sujetos mostrarán un control menos eficaz que en español; b) los sujetos con dominio básico en este idioma mostrarán un control microestructural más eficaz que el macroestructural y; c) cuando el dominio del inglés aumente, las diferencias en el CC entre inglés y español tenderán a desaparecer. Los resultados apoyaron parcialmente las predicciones: el control macroestructural en 
sujetos con dominio avanzado del inglés no fue tan eficaz como se esperaba. Además, en español la eficacia en el control macroestructural fue mayor que en el control microestructural. Los resultados fueron explicados en términos del modelo cognitivo asumido.

Palabras Clave: Comprensión de textos científicos, control de la comprensión, microestructura y macro-estructura, nivel de inglés, inglés como L2.

\begin{abstract}
We present a study with Spanish university students with elementary, intermediate and advanced English proficiency levels. We aimed at analyzing the comprehension monitoring (CM) effectiveness in English, compared to the one in Spanish, and explaining the results using the model for text comprehension developed by Kintsch and colleagues. Our focus was the Semantic mental representation, differentiating CM at micro and macro-structural levels. Micro and macro-structural CM was measured assuming the 'Error Detection Paradigm': inconsistencies were embedded in the texts and students were asked to assess the comprehensibility of them using a key-code to distinguish 'unknown words', 'absurd ideas' and 'inconsistent or contradictory ideas'. We used science texts, three in English and three in Spanish to improve reliability. Predictions were: a) in English, participants would exhibit lower efficacy in their CM than in Spanish; b) subjects with elementary English proficiency would show higher efficacy in their microstructural CM than in their macrostructural one, and; c) as English proficiency increased, the Spanish/English differences in CM would tend to disappear. Results partially supported our predictions: macrostructural CM did not improve as much as expected in subjects with advanced English proficiency levels. Unexpectedly, in Spanish, subjects tended to monitor their micro-level comprehension in a more effective way than their macro-level comprehension. These results were explained using the assumed cognitive model.
\end{abstract}

Key Words: Comprehension of scientific texts, comprehension monitoring, microstructure and macrostructure, English proficiency level, English as L2.

\title{
INTRODUCCIÓN
}

Los investigadores están de acuerdo en que leer en una segunda lengua (L2) presenta diferencias lingüísticas, de procesamiento, cognitivas, educativas, socioculturales, institucionales, respecto a leer en la lengua materna (Carrell \& Grabe, 2002; Grabe \& Stoller, 2002; Grabe, 2009).

Desde que Alderson (1984) planteara si la lectura en L2 es un problema de lectura o es un problema lingüístico, la investigación sobre lectura en segundas lenguas (Bernhardt \& Kamil, 1995; Koda, 2005) ha sugerido que la comprensión lectora en L2 es una suma de dos componentes en interacción: a) el conocimiento del propio idioma L2 a nivel léxico, sintáctico y situacional; y b) las estrategias de procesamiento 
de textos, ya desarrolladas en L1 y que son de naturaleza independiente de la lengua que se trate (Alderson, 1984; Carrell, 1991; Fukkink, Hulstijn \& Simis, 2005). En esta concepción, las estrategias son una especie de 'herramientas de gestión' del conocimiento capaces de manejar de un modo similar cualquier input lingüístico y pueden ser transferidas, en mayor o menor grado, de una lengua a otra (Cummins, 1991), aunque quizás esto suceda solo cuando se supere un cierto nivel de dominio lingüístico (Clarke, 1980).

Si las estrategias lectoras se pueden transferir de una lengua a otra, resulta interesante analizar el modo en que este proceso tiene lugar, y qué factores influyen en el además del dominio de la lengua meta L2. Centraremos nuestra atención en una de tales estrategias lectoras: el Control de la Comprensión.

\section{Marco teórico}

\subsection{El Control de la Comprensión en L2}

El Control de la Comprensión durante la lectura (CC en adelante) es una estrategia metacognitiva y se define como la habilidad de un sujeto para ser consciente, mientras lee, de si el texto tiene sentido o no lo tiene, o de si presenta inconsistencias o no (Flavell, 1981). En L1, un buen control de la comprensión se ha asociado con una buena comprensión lectora (Zabruky \& Ratner, 1986; Campanario \& Otero, 2000) y con un buen rendimiento académico (Wang, Haertel \& Walberg, 1993). Sin embargo no existe mucha investigación dedicada a estudiar el control de la comprensión lectora en L2. Como afirmaba Casanave (1988), el estudio del control de la comprensión en L2 es algo esencial pero olvidado en investigación. En los estudios que se han centrado en el CC en L2, dos son las metodologías utilizadas principalmente. Una se basa en protocolos think aloud para evaluar las reflexiones metacognitivas de los estudiantes al leer fragmentos del texto (Block, 1992). Los resultados se obtienen a partir de la categorización y análisis cualitativos posteriores de las alocuciones de los sujetos. La otra metodología usada con mucha frecuencia es el Error Detection Paradigm (Winograd \& Johnston, 1982), basada en la inserción de inconsistencias en los textos para analizar si los estudiantes las detectan o no. Estos estudios permiten análisis cuantitativos de las acciones de los sujetos, es decir, de la habilidad de los sujetos de detectar el error y corregirlo.

Algunos estudios han utilizado diseños entre-sujetos (Block, 1986), tomando un solo idioma como L1 para un grupo de sujetos y como L2 en otro grupo, lo cual involucra usualmente una gran variabilidad en variables no controladas respecto a los diseños intra-sujetos empleados en otros estudios (Morrison, 2004; Han \& Stevenson, 2008) en los cuales se toman medidas de CC en ambos idiomas, L1 y L2, lo que mejora el control de variables intrapersonales y disminuye la varianza de error. El rendimiento en L1 se toma como referencia para valorar el que se demuestra en L2. El problema metodológico de estos últimos estudios basados en detección 
de inconsistencias es que, usualmente, las instrucciones dadas a los participantes les alientan a convertir la tarea en una 'búsqueda de errores en el texto', lo cual modifica sustancialmente el propósito habitual de lectura, que es la comprensión.

Aunque en ocasiones resulta difícil explicar los resultados de esos estudios en términos cognitivos, bien por la excesiva variabilidad en la muestra, bien por la ausencia de un modelo de procesamiento determinado, o por la imposibilidad de asociar la detección de los errores insertados con niveles de procesamiento y control concretos, estos estudios han obtenido resultados convergentes: a) el control de la comprensión es más efectivo en L1 que en L2 (Block, 1986, 1992; Morrison, 2004; Han \& Stevenson, 2008); b) cuanto mayor es el nivel de dominio de L2, más efectivo es el CC en ese idioma (más similar al que se tiene en L1) (Block, 1992); y, más importante para nuestro propósito, c) los investigadores establecen una relación entre una mayor o menor efectividad en el CC en L2 y diferentes modos de procesar la información. Estas explicaciones en términos cognitivos de las diferencias entre L1 y L2 en lectura comenzaron hace tiempo. Segalowitz, Poulsen y Komoda (1991), trabajando con bilingües en francés e inglés, encontraron que los problemas de lectura en L2 se situaban en el nivel de reconocimiento de palabras y en el de las relaciones locales entre proposiciones (formación de microestructura), en lugar de situarse en el nivel de integración de la información en grandes fragmentos de texto, o entre ideas del texto y el conocimiento previo. Yamashita (2002), en un estudio sobre transferencia de habilidades lectoras del japonés (L1) al inglés (L2), encontró que sus estudiantes podían transferir sus habilidades lectoras solamente a nivel local (léxico y sintáctico) pero no a nivel global (del texto como un todo). De forma convergente, Tsai, Ernst y Talley (2010) estudiaron las estrategias en comprensión lectora en L1 (chino mandarín) y L2 (inglés) usando un test estandarizado de estrategias. Encontraron que los sujetos tendían a transferir a L2 su conocimiento lector en L1 a nivel de palabra en vez de hacerlo a niveles más altos (nivel de ideas o de todo el texto).

\subsection{Diferencias en el procesamiento de la información textual en $L 1$ y en L2}

Cuando se utiliza el Error Detection Paradigm para valorar el CC, significa detectar las inconsistencias de un texto, es decir, detectar que dos proposiciones textuales son mutuamente excluyentes y que no pueden ser ciertas a la vez. Para eso, ambas proposiciones deben procesarse a la vez en la memoria de trabajo. Vosniadou, Pearson y Rogers (1988), en un estudio realizado con niños, descubrieron que las dificultades en la detección de inconsistencias en L1 no se debían a la incapacidad de los participantes para comparar proposiciones contradictorias, sino a una pobre representación mental de una o de ambas proposiciones en la memoria. Cuando se daban las condiciones para que ambas proposiciones estuvieran a la vez en la memoria de trabajo, los niños detectaban la contradicción sin problemas.

Entonces, la pregunta relevante es: ¿cuáles son las dificultades que los sujetos pueden encontrar a la hora de representar proposiciones de un texto? Una de esas dificultades 
tiene que ver con el dominio del idioma y la automatización de los procesos más bajos de procesamiento. En L1 Perfetti (1985) propuso la Verbal Efficiency Hypothesis según la cual, automatizar los procesos de bajo nivel, tales como los procesos de acceso pre-léxico (acceso léxico, reconocimiento de palabras) y post-léxico (sintaxis, parsing) deja libres los recursos atencionales necesarios en la memoria para que los procesos de comprensión de nivel más alto tengan lugar (Graesser, Hoffman \& Clark, 1980), como es el caso de construir macroideas y establecer la coherencia global del texto. Cuando los procesos de bajo nivel no se hayan automatizado, el desempeño será ineficiente y más lento en estas operaciones básicas (descodificación de palabras desconocidas, conocimiento de vocabulario y sintaxis, etc.) y eso limitará la aplicación de procesos de alto nivel (asignar significado a cláusulas, realizar inferencias, etc.).

Como hipótesis de trabajo puede asumirse que los sujetos adultos con bajos niveles de L2 procesan esa lengua de forma similar a como lo hacen los niños que aún no han adquirido un buen dominio de su lengua materna (Segalowitz et al., 1991). Es de esperar, entonces, que los sujetos con bajo dominio en L2 tiendan a procesar los textos en esa lengua a partir de componentes de bajo nivel, como palabras o, quizás, cláusulas, porque no tienen automatizados los procesos preléxico y/o post-léxico (Fukkink et al., 2005; Taguchi, Gorsuch \& Sasamoto, 2006; Koda, 2007). Cuando el acceso léxico sea dificultoso, los sujetos estarán obligados a detenerse en cada palabra del texto y a ayudarse de la estrategia de la traducción a la lengua materna con bastante frecuencia para acceder a su significado (Gómez, Solaz \& Sanjosé, 2012). Cuando no se tenga automatizado el procesamiento post-léxico, los sujetos deberán aplicar conscientemente las reglas gramaticales para relacionar palabras entre sí, formar cláusulas y finalmente, microproposiciones en L2. La falta de automatización significa que las microproposiciones serán resultado (output) de una construcción consciente en la memoria de trabajo a partir de las palabras leídas en L2, de sus correspondientes términos en L1 y de las reglas gramaticales (input). La sobrecarga de la memoria de trabajo hará difícil que el sujeto pueda aplicar macroreglas (supresión, selección, generalización y construcción según van Dijk (1980) para construir la macro-estructura textual (Machida, 2011)). En cambio, cuando se lee en L1 y los sujetos procesan automáticamente a niveles pre-léxico y post-léxico, las microproposiciones son construidas a partir del código escrito sin trabajo consciente y la memoria de trabajo se utiliza para construir macroproposiciones (output) a partir de microproposiciones (input).

En resumen, esperamos que los sujetos con bajos niveles en L2 tengan dificultades para construir la macro-estructura textual cuando lean en esa lengua, pero no la tengan cuando lean en L1. Esos sujetos podrían, no obstante, procesar microideas y construir la microestructura textual en L2 (relaciones locales entre ideas adyacentes). Es de esperar que, a medida que el nivel de dominio de L2 crezca, la macroestructura textual en esa lengua sea cada vez más accesible y las diferencias L1/L2 vayan desapareciendo. 


\subsection{Objetivos e hipótesis}

Asumiremos en este trabajo el modelo de procesamiento de textos propuesto por Kintsch y van Dijk (1978), desarrollado luego para dar lugar al modelo ConstrucciónIntegración (Kintsch, 1988). Estos autores proponen al menos tres niveles de elaboración de una representación mental de un texto: nivel Superficial, léxica o de palabra, Base del Texto o nivel semántico y Modelo de la Situación o nivel referencial. En la Base del texto los sujetos deben construir la micro-estructura y la macro-estructura del texto. En el Modelo de la Situación el contenido semántico del texto debe vincularse al conocimiento previo mediante inferencias. Otero y Graesser (2001) concluyeron que los lectores controlaban su comprensión en estos mismos tres niveles de representación mental.

En este trabajo concentraremos la atención en la representación mental semántica, diferenciando entre niveles micro y macro-estructurales. Estudiaremos si lectores expertos en L1, pero con variados niveles de dominio del inglés, según el Marco Común Europeo de Referencia para Lenguas ${ }^{1}$ (Council of Europe, 2001), controlan su comprensión de microideas y de macroideas en esa lengua extranjera (L2) con una eficacia estratégica similar o no a la que exhiben en español (L1).

Tomando en cuenta todos estos condicionantes, y en términos de los razonamientos basados en el procesamiento de textos anteriormente expuestos, podemos formular las siguientes hipótesis:

H1: "Los universitarios mostrarán un control de su comprensión a nivel léxico de eficacia similar en español o inglés. Estos sujetos simplemente detectarán las palabras cuyo significado no conozcan y cuando el dominio del inglés crezca, el número de palabras desconocidas decrecerá".

H2: "Los participantes con dominio básico del inglés mostrarán mayor eficacia en su control de la comprensión a nivel microestructural que a nivel macroestructural cuando lean en esa lengua. Los participantes con dominio avanzado de inglés no presentarán esas diferencias en control entre niveles micro y macroestructural”.

H3: “Los participantes con dominio básico del inglés mostrarán menor eficacia en su control de la comprensión en inglés que en español, tanto a nivel microestructural como macroestructural. Sin embargo, los participantes con dominio avanzado del inglés no presentarán esas diferencias entre idiomas".

En español (L1), no son esperables diferencias en el control micro y macroestructural en nuestros sujetos, que debería ser muy eficaz en ambos niveles. Por tanto, podemos formular la siguiente hipótesis:

H4: "En sujetos con dominio básico del inglés, las diferencias entre niveles macro y microestructural en la eficacia del control de la comprensión en inglés, serán de 
mayor magnitud que esas diferencias en español. Esto no sucederá en los sujetos con dominio avanzado del inglés".

En su estudio precedente sobre comprensión de textos académicos en inglés de universitarios cuya lengua materna es el español, Ibáñez (2008) ya encontró que el nivel de dominio de la lengua extranjera fue el predictor más importante. Por tanto, para contrastar las hipótesis anteriores tomaremos sujetos universitarios nativos españoles con variado dominio del inglés y usaremos textos expositivos en tareas de comprensión lectora para valorar su control de la comprensión. Los universitarios han demostrado un alto nivel de comprensión lectora y deben haber desarrollado un CC alto en L1 que podrían transferir a L2. Usaremos inconsistencias insertadas en los textos evitando alterar el propósito de lectura, como se explicará después.

\section{Marco Metodológico}

\subsection{Participantes}

Participaron en la investigación 156 estudiantes universitarios pero obtuvimos datos completos de 153 estudiantes todos ellos españoles y de ambos sexos: 118 de ellos se encontraban en segundo año de pregrado (20-25 años) y 35 postgraduados titulados en Filología Inglesa. Los estudiantes de pregrado pertenecían a 5 grupos naturales en una facultad de formación de profesores de educación infantil y primaria. Este grado universitario incluye materias de contenido específico (ciencias, matemáticas, historia, etc.), lenguas (español y una lengua extranjera, predominantemente inglés) y ciencias de la educación (pedagogía, psicología de la educación y del desarrollo, didácticas específicas, prácticas de enseñanza). Todos los participantes demostraron un dominio del español al nivel C1 o superior, como requisito de acceso a los estudios universitarios en España. Además, todos ellos estudiaron inglés como lengua extranjera. Los postgraduados Filólogos pertenecían a un grupo natural en un Máster Universitario para ser profesores de Educación Secundaria de inglés.

\subsection{Diseño y variables}

Las variables dependientes consideradas fueron el CC en español y también en inglés. Estas medidas se obtuvieron a través de una tarea de lectura que se describe después. Obtuvimos puntuaciones de CC en el nivel de palabra, en el nivel microestructural y en el nivel macroestructural.

El nivel de dominio del inglés se consideró un factor independiente en los análisis. Todos los sujetos demostraron un nivel C1 o C2 en su lengua materna (español) para poder acceder a la universidad española.

\subsection{Materiales y medidas}

Utilizamos varios instrumentos para obtener las medidas necesarias. 


\subsubsection{Materiales de medición}

A) Dominio del inglés. La puntuación del test estandarizado 'the Oxford Online Placement Test' (OOPT, http:// www. oxfordenglishtesting. com http://fds.oup. com/www.oup.com /elt/oet/ift/oopt_measure.pdf) se utilizó para determinar tres grandes niveles de dominio del inglés como lengua extranjera, según el Marco Común Europeo de Referencia para Lenguas: básico (A1-A2), intermedio (B1-B2) o avanzado (C1-C2). Los postgraduados se situaron todos ellos en el grupo de dominio avanzado del inglés, mientras los estudiantes de pregrado se dividieron entre los niveles de dominio básico e intermedio. Las medias respectivas de los tres grupos de sujetos considerados en el test OOPT fueron: $\mathrm{M}_{\text {básico }}=26.7$ (DT=9.4); $\mathrm{M}_{\text {intermedio }}=54.5$ (DT= 9.1); $\mathrm{M}_{\text {avanzado }}=81.4(\mathrm{DT}=6.7)$. Estos valores fueron claramente diferentes con una alta significación y un tamaño del efecto muy grande $\left(\mathrm{F}(2,150)=362.487 ; \mathrm{p}<.001 ; \eta^{2}=\right.$ .83). Comparaciones por pares realizas post-hoc (Bonferroni) mostraron diferencias significativas entre cada par de niveles con una confianza del 99\% o superior.

B) Control de la Comprensión lectora. Asumimos el Error Detection Paradigm para relacionar el CC con la capacidad para detectar inconsistencias insertadas en varios textos en español e inglés.

\subsubsection{Materiales de interacción}

Preparamos 6 textos expositivos sobre temas generales de ciencias experimentales, 3 de ellos en español y 3 en inglés. Los textos expositivos como los de ciencias, a diferencia de los narrativos, suelen estar libres de ambigüedades lo cual debe evitar que los lectores atribuyan las inconsistencias a licencias literarias. Los textos fueron tomados de pruebas de acceso a la universidad en España, y luego fueron adaptados para los propósitos del experimento. Para asegurar la fiabilidad, controlamos las siguientes variables en los textos: extensión, dificultad lectora en inglés y similitud entre los textos en español y los textos en inglés.

Los textos contuvieron entre 210 y 250 palabras y se estructuraron en 3 secciones o párrafos: el primero donde se introduce el tema, el segundo de desarrollo de algunos aspectos de ese tema, y el último de resumen con las ideas principales (macroideas) del texto. La dificultad lectora de los 3 textos en inglés se ajustó por un doble procedimiento. Primero se consideraron 10 posibles textos en inglés y se utilizó la escala Flesch-Kincaid Readability Scale (www.readabilityformulas.com) para fijar su dificultad entre 60 y 70 puntos, (que corresponde al nivel de $8^{\circ}-9^{\circ}$ grado en nativos ingleses). A continuación, 2 expertos profesores de lengua inglesa categorizaron esos mismos textos según los niveles dados por el marco de referencia europeo para las lenguas. Para el experimento seleccionamos 6 textos que se clasificaron por ambos jueces en los niveles A2 o B1. A continuación, 3 de los 6 textos se eligieron al azar y se tradujeron al español para asegurar en lo posible la igualdad en el diseño de los textos en ambos idiomas. Una nativa británica bilingüe revisó los 3 textos en inglés para mejorar y adaptar algunas expresiones idiomáticas. 
Los textos se modificaron para introducir 2 inconsistencias microestructurales y 2 inconsistencias macroestructurales. Denominamos 'frases diana' a las frases que contenían alguna de las inconsistencias insertadas. Las inconsistencias microestructurales consistieron en adjetivos calificativos impropios del sustantivo que acompañaban (por ejemplo, hot ice). Las inconsistencias macroestructurales consistieron en frases explícitamente contradictorias con ideas importantes y anteriores en el texto. Los lectores no necesitaban activar ideas externas al texto para detectar esas inconsistencias macroestructurales. Es importante notar que la comprensión del contenido de los textos dependía de la extracción de las macroideas de los mismos, dos de las cuales fueron contradichas. Por tanto, el control macroestructural se asoció directamente con la construcción de la macroestructura de los textos.

El Anexo recoge uno de los textos en inglés utilizado en el experimento. En el pueden verse las inconsistencias micro y macroestructurales introducidas (subrayadas para su localización).

\subsubsection{Medidas}

Para saber qué tipo de inconsistencias detectaron los estudiantes, en las instrucciones se les proporcionó una clave para categorizar la información subrayada. Los participantes debían usar el siguiente código: '1' debajo de la información absurda o sin sentido (caso de las inconsistencias micro-estructurales); '2' para la información no coherente o contradictoria con otras ideas en el texto (caso de las inconsistencias macro-estructurales) y; '3' para las palabras desconocidas en cualquier lugar del texto (ver Anexo). Algunos participantes subrayaron palabras desconocidas dentro de una 'frase diana' y usaron el código correspondiente ('3') en lugar del esperado según la inconsistencia insertada (códigos ' 1 ' o '2'). En estos casos, el CC realizado por el sujeto no fue el esperado (de nivel semántico), pero representó una clase de CC a nivel léxico. Estos casos hicieron ver la conveniencia de utilizar medidas conservadoras para el CC, basadas en la ausencia de cualquier tipo de subrayado en lugar de utilizar medidas basadas directamente en el número de subrayados realizados.

Por tanto, consideramos las siguientes medidas CC:

1. Número total de palabras subrayadas como 'desconocidas' en cualquier frase del texto (no solo en las 'frases diana'). Los participantes necesitaron señalar palabras con significado desconocido que les impidieron comprender ideas del texto. Por tanto, esta medida está relacionada con el conocimiento de vocabulario y mide el control de la comprensión a nivel léxico.

2. Ausencia de un adecuado CC microestructural en cada idioma. Su valor puede variar entre 0 y 6 puesto que se leyeron 3 textos en cada idioma, conteniendo 2 inconsistencias microestructurales cada uno.

3. Ausencia de un adecuado CC macroestructural en cada idioma, cuyo valor puede variar también entre 0 y 6 . 
Las variables definidas en los puntos 2 y 3 anteriores varían inversamente con la eficacia en el control de los sujetos: a mayor puntuación, menos eficaz es el control de la comprensión.

Finalmente, las instrucciones y los 6 textos experimentales fueron sometidos al juicio de dos sujetos que no participaron en la muestra. Como resultado, se mejoró la comprensibilidad de las instrucciones y algunas inconsistencias microestructurales introducidas en los textos fueron sustituidas por otras.

\subsection{Diseño experimental}

El diseño experimental fue factorial mixto, 2 X 2 X 3, con 2 factores intrasujeto, 'Idioma' (L2: inglés/ L1: español) y 'nivel de Control de la Comprensión' (micro/ macroestructural) y 1 factor entre-sujetos, el 'Dominio del Inglés' (básico/ intermedio/ avanzado).

\subsection{Procedimiento}

Los estudiantes participaron voluntariamente en el experimento. Las puntuaciones del dominio del inglés se obtuvieron en una sesión previa. Los estudiantes completaron el test (OOPT) individualmente en el ordenador en un tiempo entre 30 y 50 minutos.

La sesión experimental se presentó como una investigación dedicada a mejorar los textos de educación científica, ya que suelen ser difíciles de entender para muchos estudiantes. Se repartieron a los participantes las instrucciones por escrito y un ejemplo para practicar antes de comenzar con los textos. Uno de los investigadores leyó las instrucciones y ejecutó el ejemplo de práctica en voz alta dando con todas las explicaciones necesarias para asegurar la comprensión de la tarea demandada y el uso del código. Este código se debía mantener a la vista para su consulta cuando fuera necesario (ver Anexo).

Se aprovechó la vocación de los participantes como maestros de primaria y profesores de secundaria para pedirles que juzgaran la comprensibilidad de los textos de ciencias a las posibilidades de aprendizaje de sus futuros estudiantes. De un modo deliberado y explícito, en las instrucciones se advirtió que podrían encontrar diferentes obstáculos de comprensión, inconsistencias, contradicciones, información absurda o palabras sin sentido dependiendo del criterio, de las estrategias de lectura y del conocimiento de cada participante. De este modo la tarea para evaluar el CC no consistió en 'una búsqueda de errores', como sucede en otras investigaciones, lo cual modifica sustancialmente el propósito habitual de lectura, que es la comprensión.

Se resolvieron las dudas de los participantes antes de repartir los textos. La presencia de textos en inglés fue justificada por la reciente oferta para la integración de España en el Espacio Europeo de Educación Superior (http://www.ehea.info/). 
Los 3 textos en inglés (lengua extranjera) se repartieron en orden contrabalanceado, y siempre antes que los 3 textos en español. De este modo se evitó que la esperada eficaz detección de inconsistencias en español creara una alerta espuria sobre los textos en inglés y, por tanto, alterara las condiciones usuales de lectura de cada participante. No se limitó el tiempo para cada texto y la tarea completa requirió menos de 50 minutos.

Los datos fueron analizados mediante ANOVA considerando en cada caso los factores implicados en el diseño y las hipótesis a contrastar. Se procuró aportar datos, no solo del nivel de confianza para rechazar la hipótesis nula (significación), sino también del tamaño del efecto (eta cuadrado parcial, $\eta^{2}$ ) y su interpretación según Cohen, que está relacionado con la potencia estadística.

\section{Resultados}

\subsection{CC a nivel léxico}

Aunque el comportamiento del CC a nivel de palabra es predecible, dado que los sujetos subrayaron palabras cuyo significado les era desconocido, contabilizamos el número de palabras subrayadas por los estudiantes en cualquier parte del texto. Los sujetos detectaron más palabras de significado desconocido en inglés $\left(\mathrm{M}_{\text {lex }}\right.$ inglés $=7.4$ $(\mathrm{DT}=6.8))$ que en español $\left(\mathrm{M}_{\text {lex }}\right.$ español $\left.=1.1(\mathrm{DT}=1.1)\right)$ con un efecto significativo y de tamaño grande del idioma $\left(\mathrm{F}(1,150)=170.951 ; \mathrm{p}<.001 ; \eta^{2}=.53\right)$, un efecto significativo del dominio del inglés $\left(\mathrm{F}(2,150)=37.032 ; \mathrm{p}<.001 ; \eta^{2}=.33\right)$ y también una interacción significativa entre ambos factores $\left(\mathrm{F}(2,150)=36.570 ; \mathrm{p}<.001 ; \eta^{2}=.33\right)$, debida a que las palabras desconocidas en inglés disminuyeron mucho a medida que aumentó el dominio en ese idioma, pero el número de palabras desconocidas en español apenas cambió. El número de palabras detectadas en L1 fue pequeño y focalizado en términos científicos, mientras que en L2 se relacionó con su nivel de dominio del inglés. Los sujetos con dominio básico e intermedio del inglés subrayaron un número importante de palabras desconocidas en los tres textos $\left(\mathrm{M}_{\text {ba-lex }}=12.74(\mathrm{DT}=8.10) ; \mathrm{M}_{\text {interm-lex }}=\right.$ $7.41(\mathrm{DT}=5.16))$. Los sujetos con dominio avanzado del inglés subrayaron un número menor de palabras desconocidas $\left(\mathrm{M}_{\text {avan-lex }}=1.43(\mathrm{DT}=1.29)\right)$ como cabía esperar. Las diferencias fueron significativas $(\mathrm{F}(2,150)=38.114 ; \mathrm{p}<.001)$ y el tamaño del efecto fue grande $\left.\eta^{2}=.34\right)$.

\subsection{Sobre diferencias entre control microestructural y control macroestructural en inglés (en relación con la hipótesis $\mathrm{H2}$ )}

La hipótesis H2 implica la predicción de diferencias significativas entre las medidas de control micro y macroestructural en inglés, en sujetos con dominio bajo de esta lengua, pero no en sujetos con dominio alto de inglés. Es decir, la $\mathrm{H} 2$ predice una interacción entre el factor 'nivel de control' (micro/ macroestructural) y el factor 'dominio del inglés'. 
Los participantes con dominio básico de inglés presentaron mayor número de inconsistencias no detectadas en inglés a nivel macroestructural $\left(\mathrm{M}_{\text {básico-macro }}=4.32\right.$ $(\mathrm{DT}=1.44))$ que a nivel microestructural $\left(\mathrm{M}_{\text {básico-micro }}=3.32(\mathrm{DT}=1.19)\right)$ con un tamaño del efecto grande $\left(\mathrm{F}(1,37)=14.060 ; \mathrm{p}=.001 ; \eta^{2}=.28\right)$. Es decir, el control macroestructural en inglés de estos sujetos fue significativamente menos eficaz que su control a nivel microestructural.

Por su parte, los participantes con dominio intermedio de inglés presentaron mayor número de inconsistencias macroestructurales no detectadas en inglés $\left(\mathrm{M}_{\text {interm- }}\right.$ $\left.{ }_{\text {macro }}=3.81(\mathrm{DT}=1.30)\right)$ que de inconsistencias microestructurales no detectadas en inglés $\left(\mathrm{M}_{\text {interm-micro }}=2.69(\mathrm{DT}=1.37)\right)$ con un tamaño del efecto grande $(\mathrm{F}(1,79)=$ 40.245; $\left.\mathrm{p}<.001 ; \eta^{2}=.34\right)$. Es decir, el control macroestructural en inglés de estos sujetos también fue significativamente menos eficaz que su control microestructural.

Finalmente, los participantes con dominio avanzado del inglés también presentaron significativamente mayor número inconsistencias macroestructurales no detectadas en inglés $\left(\mathrm{M}_{\text {avan-macro }}=3.09(\mathrm{DT}=1.48)\right)$ que microestructurales $\left(\mathrm{M}_{\text {avan-micro }}=\right.$ 1.40 (DT=1.33)) con un tamaño del efecto muy grande $(\mathrm{F}(1,34)=60.882 ; \mathrm{p}<.001$; $\eta^{2}=$.64). Es decir, también el control macroestructural en inglés de estos sujetos fue significativamente menos eficaz que su control a nivel microestructural, de un modo cualitativamente similar a lo que sucedió en los grupos de dominio de inglés básico e intermedio.

Un ANOVA tomando los tres grupos de participantes conjuntamente reveló un efecto principal significativo del factor 'dominio del inglés' (básico/intermedio/ avanzado) con un tamaño del efecto grande $\left(\mathrm{F}(2,150)=18.834 ; \mathrm{p}<.001 ; \eta^{2}=.20\right)$. El efecto del factor intra-sujetos, 'nivel del control' (micro/macroestructural) es también significativo y con tamaño del efecto grande $\left(F(1,150)=91.236 ; \mathrm{p}<.001 ; \eta^{2}=.38\right)$. Lo más interesante es que no hay efecto de interacción significativa entre ambos factores 'nivel de control' $X$ 'dominio del inglés' $(F(2,150)=2.149 ; \mathrm{p}>$.10) lo que significa que las diferencias micro/macro encontradas en cada nivel de dominio de inglés, no son significativamente diferentes entre sí. El Gráfico 1 presenta estos efectos. 


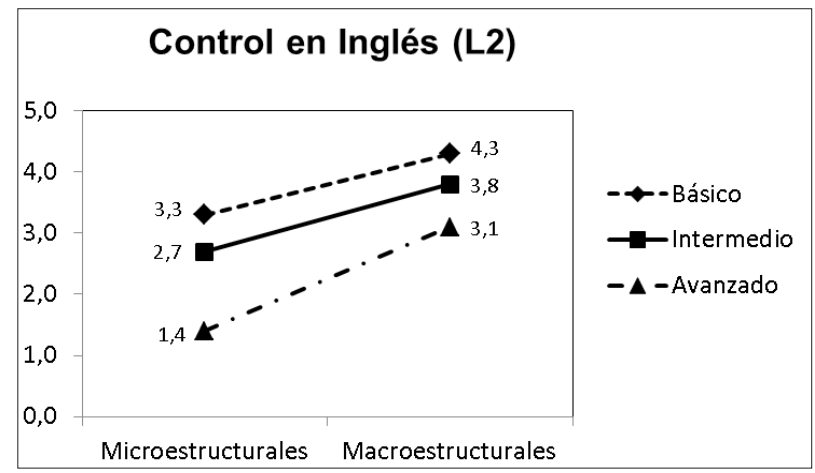

Gráfico 1. Diferencias entre control microestructural y macroestructural en inglés (L2) en los tres niveles de dominio del inglés considerados. Los valores corresponden a ausencia de detección de inconsistencias.

\subsection{Sobre diferencias entre inglés y español en control microestructural y en control macroestructural (en relación con la hipótesis $\left.\mathrm{H3}_{3}\right)$}

Desde el punto de vista estadístico la H3 implica la predicción de un efecto de interacción entre el factor 'idioma' (inglés/español) y el factor 'dominio de inglés' en las medidas asociadas al control de la comprensión de las microideas y de las macroideas.

En lo que respecta al control a nivel microestructural, los sujetos con dominio básico de inglés mostraron mayor efectividad en esta destreza en español $\left(\mathrm{M}_{\text {bas }}\right.$ español= $1.95(\mathrm{DT}=1.47))$ que en inglés $\left(\mathrm{M}_{\text {bas }}\right.$ inglés $\left.=3.32(\mathrm{DT}=1.19)\right)$ y las diferencias fueron significativas con un tamaño del efecto grande $\left(\mathrm{F}(1,37)=17.545 ; \mathrm{p}<.001 ; \eta^{2}=.32\right)$. Lo mismo sucedió con los sujetos de dominio intermedio del inglés $\left(M_{\text {interm }}\right.$ inglés $=2.69$ $\left.(\mathrm{DT}=1.37) ; \mathrm{M}_{\text {interm }}^{\text {español }}=1.56(\mathrm{DT}=1.30) ; \mathrm{F}(1,79)=49.147 ; \mathrm{p}<.001 ; \eta^{2}=.38\right)$. Sin embargo, los sujetos de dominio avanzado del inglés no mostraron diferencias entre ambos idiomas, como se esperaba $\left(\mathrm{M}_{\text {avan }}\right.$ inglés $=1.40(\mathrm{DT}=1.33) ; \mathrm{M}_{\text {avan }}$ español $=1.14$ (DT= 1.40); $\mathrm{F}(1,34)=1.000 ; \mathrm{p}=.324)$. El Gráfico $2 \mathrm{~A}$ muestra estos resultados. 


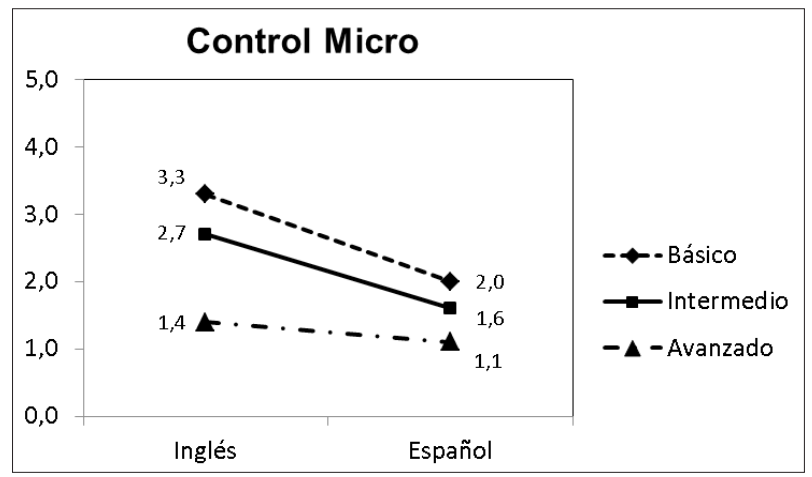

Gráfico 2A. Diferencias en el control microestructural entre inglés (L2) y español (L1) en los tres niveles de dominio del inglés considerados. Los valores corresponden a ausencia de detección de inconsistencias en microideas.

Los sujetos de dominio básico del inglés presentaron diferencias significativas $\left(\mathrm{F}(1,37)=128.571 ; \mathrm{p}<.001 ; \eta^{2}=.78\right)$ en su control macroestructural cuando leen en inglés $\left(\mathrm{M}_{\text {bas }}\right.$ inglés $\left.=4.32(\mathrm{DT}=1.44)\right)$ respecto a cuando leen en español $\left(\mathrm{M}_{\text {bas }}{ }^{\text {español }}=1.16\right.$ $(\mathrm{DT}=1.20))$. El tamaño del efecto fue grande. Lo mismo sucedió con los sujetos de dominio intermedio del inglés $\left(\mathrm{M}_{\text {interm }}\right.$ inglés $=3.81(\mathrm{DT}=1.30) ; \mathrm{M}_{\text {interm }}$ español $=0.79$ (DT= $\left.0.98) ; \mathrm{F}(1,79)=289.232 ; \mathrm{p}<.001 ; \mathrm{h}^{2}=.79\right) \mathrm{y}$, en contra de lo esperado, también con los sujetos de dominio avanzado de inglés $\left(\mathrm{M}_{\text {avan }}\right.$ inglés $=3.09(\mathrm{DT}=1.48) ; \mathrm{M}_{\text {avan }}$ español $=1.26$ $\left.(\mathrm{DT}=1.54) ; \mathrm{F}(1,34)=54.528 ; \eta^{2}=.62\right)$. El Gráfico $2 \mathrm{~B}$ muestra estos resultados.

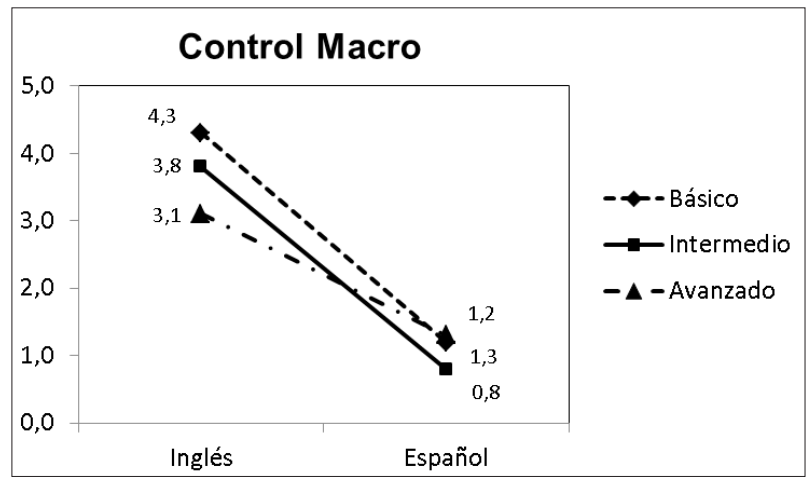

Gráfico 2B. Diferencias en el control macroestructural entre inglés (L2) y español (L1) en los tres niveles de dominio del inglés considerados. Los valores corresponden a ausencia de detección de inconsistencias en macroideas. 


\subsection{Sobre las diferencias entre control micro y macroestructural en cada uno de los idiomas L1 y L2, y su comparación (en relación con la hipótesis $\mathrm{H}_{4}$ )}

Se realizó un ANOVA tomando el idioma (inglés/español) y el nivel de control de la comprensión (micro/macroestructural) como factores intra-sujeto, y el nivel de dominio del inglés (básico/intermedio/avanzado) como factor entre-sujetos. El control en inglés fue significativamente menos eficaz que en español $(F(1,159)=345.399$; $\mathrm{p}<$ $\left..001 ; \eta^{2}=.70\right)$, hubo control significativamente más eficaz a nivel microestructural que a nivel macroestructural en general (i.e. hubo menor número de inconsistencias microestructurales no detectadas que macroestructurales: $\mathrm{F}(1,150)=17.466 ; \mathrm{p}<.001$; $\left.\eta^{2}=.10\right)$ y a medida que creció el dominio del inglés hubo menos errores de detección de inconsistencias de todo tipo $\left(\mathrm{F}(2,150)=10.753 ; \mathrm{p}<.001 ; \eta^{2}=.13\right)$. Todo ello en coherencia con lo encontrado en el contraste de $\mathrm{H} 2$ y H3.

Apareció un interesante efecto de interacción 'idioma' $\mathrm{X}$ 'nivel de control' $\left(\mathrm{F}(1,150)=76.809 ; \mathrm{p}<.001 ; \eta^{2}=.13\right)$ indicando que las diferencias en control micro/ macro de los participantes fueron de diferente magnitud en inglés y en español. En español, la cantidad de fallos de control en frases diana fue pequeño, pero el control microestructural fue significativamente menos eficaz que el control macroestructural $\left(\mathrm{M}_{\text {micro }}{ }^{\text {español }}=1.56(\mathrm{DT}=1.39) ; \mathrm{M}_{\text {macro }}\right.$ español $=.99(\mathrm{DT}=1.19), \mathrm{F}(1,152)=18.171 ; \mathrm{p}<.001$; $\mathrm{h} 2=.11)$, en contra de lo esperado. Estas diferencias se produjeron en los estudiantes de pregrado de la muestra, pero no en el subgrupo de filólogos $(\mathrm{F}<1)$.

Por tanto, las diferencias micro/macro en ambos idiomas fueron de distinto signo para los participantes con dominios básico e intermedio de inglés. En estos estudiantes, el control macroestructural fue más eficaz que el microestructural en español, pero en inglés sucedió al contrario. Y ello dio lugar al efecto significativo de interacción 'Idioma' X 'Nivel de Control' que puede ser visualizado en los Gráficos $3 \mathrm{~A}, 3 \mathrm{~B}$ y $3 \mathrm{C}$.

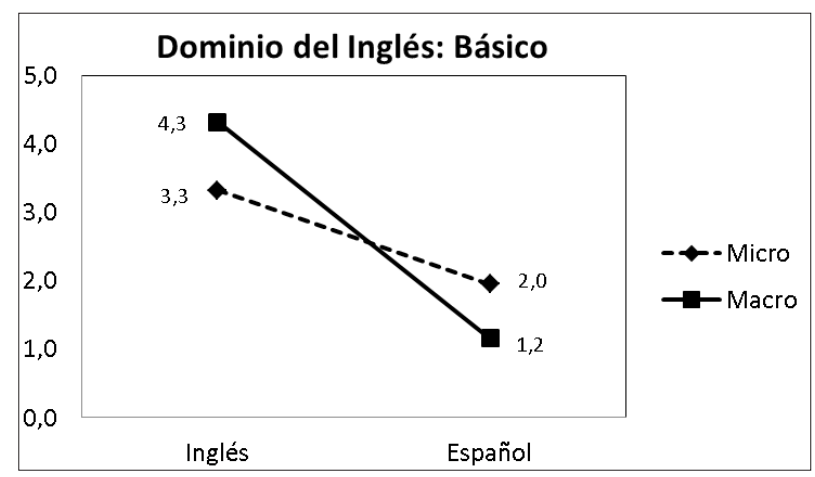

Gráfico 3A. Interacción entre el idioma (inglés/ español) y el nivel de control de la comprensión (micro/ macroestructural) para los sujetos con dominio básico del inglés. Los valores corresponden al número de inconsistencias micro o macroestructurales no detectadas (máximo 6). 


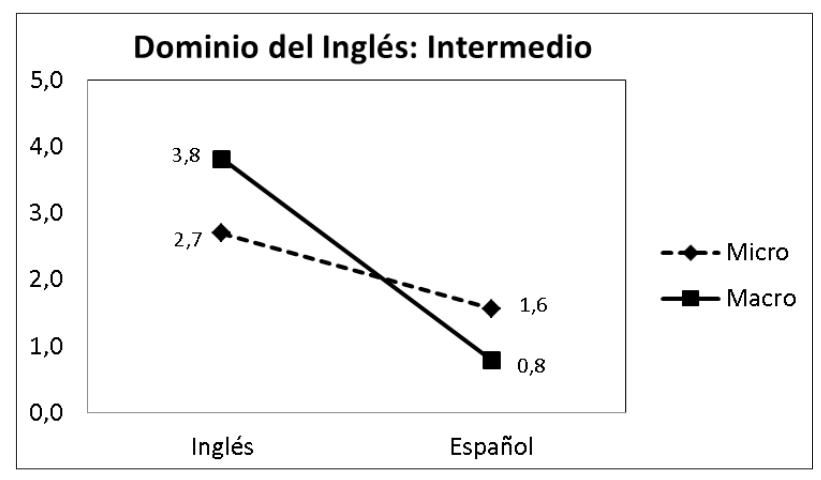

Gráfico 3B. Interacción entre el idioma (inglés/español) y el nivel de control de la comprensión (micro/ macroestructural) para los sujetos con dominio intermedio del inglés. Los valores corresponden al número de inconsistencias micro o macroestructurales no detectadas (máximo 6).

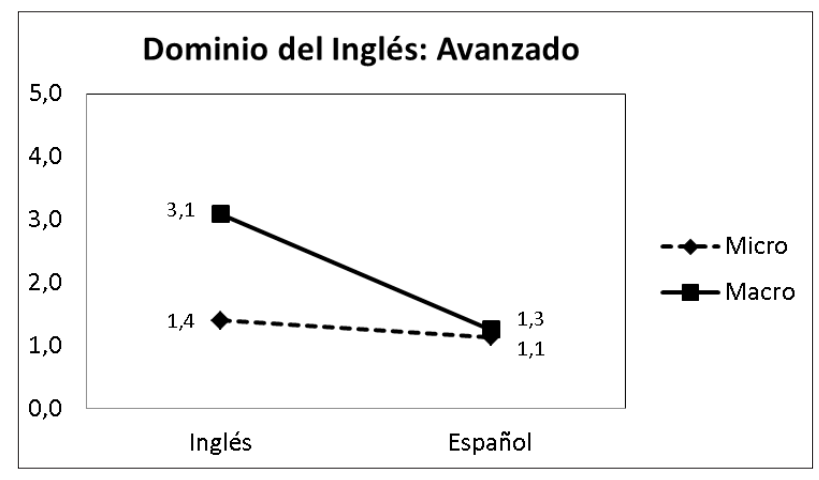

Gráfico 3C. Interacción entre el idioma (inglés/español) y el nivel de control de la comprensión (micro/ macroestructural) para los sujetos con dominio avanzado del inglés. Los valores corresponden al número de inconsistencias micro o macroestructurales no detectadas (máximo 6).

También aparecieron efectos de interacción significativos entre el factor 'dominio del inglés' y los otros dos. Así, en el grupo de sujetos con alto dominio del inglés, el control de la comprensión en ese mismo idioma mejoró significativamente respecto de los otros grupos de dominio del inglés $\left(\mathrm{F}(2,150)=13.221 ; \mathrm{p}<.001 ; \eta^{2}=.15\right)$, pero no así el control en español. En este grupo de dominio avanzado del inglés, el control microestructural fue significativamente más eficaz que en el de los otros dos grupos $\left(\mathrm{F}(2,150)=6.424 ; \mathrm{p}=.002 ; \eta^{2}=.08\right)$ aunque no sucedió así en el control macroestructural. 
Sin embargo, no apareció la interacción triple 'idioma' $\mathrm{X}$ 'nivel de control' $\mathrm{X}$ 'dominio del inglés' $(\mathrm{F}<1)$ indicando que la magnitud de la interacción 'idioma' $\mathrm{X}$ 'nivel de control' mostrado en los Gráficos 3A, 3B y $3 \mathrm{C}$ no fue significativamente diferente en los tres niveles de dominio del inglés.

\section{CONCLUSIONES}

Los resultados obtenidos a nivel superficial o léxico confirman la primera hipótesis (H1) planteada en nuestro estudio, que anticipaba que los estudiantes universitarios mostrarían un control de la comprensión adecuado en este nivel. Las diferencias encontradas en el número de palabras desconocidas se asocian claramente a un mayor o menor dominio del idioma.

En la representación semántica, micro y macroestructural, que es el foco de este trabajo, las hipótesis formuladas recibieron soporte empírico parcial. La segunda hipótesis planteada (H2) anticipaba que los estudiantes con un dominio básico del inglés mostrarían un mejor control de la comprensión a nivel microestructural que a nivel macroestructural en esa lengua, mientras que esas diferencias en entre el control micro y macroestructural desaparecían con un dominio avanzado del inglés. En efecto, el control macroestructural en inglés de los sujetos con dominio básico e intermedio del inglés fue significativamente menos eficaz que su control a nivel microestructural. Esto apoya la hipótesis H2. Sin embargo, y en contra de lo esperado en $\mathrm{H} 2$, los sujetos con nivel de dominio avanzado del inglés también mostraron un control macroestructural en inglés significativamente menos eficaz que su control microestructural, de un modo cualitativamente similar a lo que sucedió en los grupos de dominio del inglés básico e intermedio.

En cuanto a las diferencias predichas en nuestra tercera hipótesis (H3) entre L1 y L2 en la eficacia del control de la comprensión micro y macroestructural en sujetos de dominio básico del inglés, los datos sobre control microestructural mostraron diferencias significativas entre ambos idiomas en sujetos con dominio básico e intermedio del inglés. Sin embargo los sujetos con dominio avanzado del inglés mostraron una eficacia en el control microestructural en inglés similar al que mostraron en español, de acuerdo con nuestra hipótesis. Las diferencias L1/L2 en control macroestructural mostraron un patrón algo diferente. Los sujetos con dominio básico e intermedio del inglés mostraron claras dificultades en el procesamiento macroestructural en inglés comparado con su desempeño en español. Contrariamente a lo predicho, los sujetos de dominio avanzado del inglés se comportaron como los de dominio básico e intermedio, aunque sus respectivas diferencias entre L1 y L2 en el control macroestructural fueron cuantitativamente distintas. Por tanto, la hipótesis H3 fue sustentada por los datos en el control microestructural, pero parcialmente refutada en el control macroestructural. 
En resumen, el control de la comprensión en el nivel de representación semántica del texto en inglés es, globalmente, menos eficaz que en español. Esperábamos que la eficacia en el control en inglés mejorara mucho más a medida que el nivel de dominio de este idioma aumenta. En los límites de nuestro experimento esto solamente ha sucedido en el nivel microestructural, pero no en el macroestructural.

El modelo teórico asumido sugiere que los errores de control proceden de una deficiente representación de una o de ambas proposiciones incoherentes en la memoria. De acuerdo con ello, nuestros estudiantes con dominio básico o intermedio del inglés procesan textos en este idioma basándose en componentes de bajo nivel (palabras y cláusulas que corresponden a ideas elementales, o microideas). Por ello, estos sujetos representarán las macroideas del texto en su memoria de forma más pobre que las microideas en L2. En español, los textos son procesados a partir de componentes de alto nivel, frases o ideas completas, y las macroideas (así como las microideas) son representadas de forma adecuada en la memoria.

Nuestros resultados están de acuerdo con esta explicación teórica, al igual que los de Block (1992), Yamashita (2002), o Tsai et al. (2010): nuestros participantes mostraron dificultades notables para construir la macroestructura textual de forma adecuada y no detectaron muchas de esas inconsistencias. Incluso nuestros participantes con mayor dominio del inglés todavía mostraron diferencias significativas entre L1 y L2 en el control macroestructural. Una formación (y una evaluación del dominio) en lengua extranjera centrada más en la comprensión de microideas que en la de macroideas, sin prestar la suficiente atención a la comprensión de textos extensos y, por tanto, a la construcción de la macroestructura, podría explicar este resultado.

Finalmente nuestros datos muestran que los sujetos con dominio básico o intermedio del inglés, controlan su comprensión en esa lengua de forma más eficiente a nivel microestructural, pero en español el control más eficiente se produce a nivel macroestructural.

La contrastación de nuestra cuarta hipótesis (H4) hizo patente que las diferencias en control microestructural respecto del macroestructural fueron menores en español que las que se dieron en inglés. Sin embargo, y de forma inesperada, en los sujetos con dominio básico e intermedio del inglés se produjeron diferencias significativas entre control micro y macroestructural en español, en sentido inverso a las diferencias encontradas en inglés: en español las macroideas se controlaron de un modo más eficaz que las microideas. Esta diferencia no se produjo entre los sujetos de dominio avanzado del inglés, quizás porque su formación como filólogos les provee también de un dominio más avanzado del español, (el grado universitario incluye numerosas materias de formación filológica en español, además de materias en inglés propias de su especialidad). 
Aunque este efecto no esperado (control microestructural menos efectivo que control macroestructural en español) merece un estudio detallado posterior, podemos adelantar dos conjeturas razonables para explicarlo. La primera es que el mayor desarrollo de las estrategias cognitivas durante la lectura en L1 puede hacer que los estudiantes, aun detectando la inconsistencia microestructural (recordemos que se trataba de un adjetivo absurdo para el sustantivo que acompañaba), regulen su comportamiento suponiendo que se trata de una 'errata' textual. Dado que tales inconsistencias no eran importantes para el significado de las ideas importantes del texto, tal comportamiento, aun en contra de las instrucciones recibidas en el experimento, no supondría ningún problema de comprensión para los estudiantes. Nuestro instrumento de evaluación del CC únicamente consideró como acción de regulación el subrayado y codificación de las inconsistencias, significados absurdos y palabras desconocidas detectadas por los estudiantes, pero no consideró otras posibilidades de reacción ante información anómala que los estudiantes presentan (Chinn \& Brewer, 1993).

La segunda posibilidad es que la meta de los sujetos al leer en español, vinculada con el procesamiento macroestructural, suprima información poco relevante de la memoria de procesamiento e impida una representación adecuada de las microideas. Dado que las microideas absurdas insertadas en los textos experimentales son procesadas online al leer, el mecanismo de supresión debería estar asociado a una inhibición poderosa de la microidea antagónica procedente del conocimiento previo del sujeto. Otero y Kintsch (1992) usaron este mecanismo para explicar el fenómeno conocido como The Moses Illusion (Erickson \& Mattson, 1981), consistente en la no detección de la inconsistencia implicada en la pregunta: ‘Cuántas parejas distintas de animales introdujo Moisés en el Arca?'. La atención derivada hacia la respuesta a la pregunta, y una representación deficiente y poco diferenciadora entre 'Moisés' y 'Noé' en la memoria, hacen que muchas personas no detecten la inconsistencia de la frase de la pregunta. Según el modelo de procesamiento de textos conocido como 'modelo Construcción-Integración' (Kintsch, 1988), para que se detecte la inconsistencia, las dos proposiciones contradictorias implicadas deben superar un cierto umbral de activación (asociado con la consciencia) al finalizar la fase de Integración. Si una de las proposiciones contradictorias recibe mucha más activación que la otra en la fase de Construcción (por sus conexiones excitatorias con otras proposiciones activas), la primera acabará por suprimir a la segunda en la memoria de trabajo en la fase de Integración, dada la interconexión inhibitoria entre ellas. En ese caso, la inconsistencia/contradicción no se detectará. Pero si ambas proposiciones reciben una activación similar, la conexión inhibitoria actuará simétricamente y no podrá desactivar ninguna de ellas. Entonces el lector detectará la inconsistencia como consecuencia de la imposibilidad de lograr una red semántica coherente en la fase de Integración. 
Consideremos un ejemplo en el caso que nos ocupa. Un alumno lee un texto sobre el deterioro de los hábitats donde viven las distintas especies de anfibios, en donde se informa de que estos animales pueblan toda clase de hábitats en nuestro planeta. Este segmento de información incluye una de las inconsistencias insertadas a propósito, asociada con la proposición ' $\mathrm{EN}(j u n g l a$, polo)'. El estudiante podría activar una red semántica de su conocimiento previo a partir del término 'jungla' que incluyera la proposición 'EN(jungla, trópico)', inconsistente con la del texto. En la fase de Construcción, esta proposición verdadera podría llevarse a la memoria de procesamiento por su enlace con el concepto 'jungla' y relacionarse con la proposición falsa mediante una conexión inhibitoria. En el caso en que la proposición verdadera recibiera una fuerte activación a partir de sus conexiones excitatorias con otras proposiciones o conceptos del texto (por ejemplo desde 'jungla'), y que la proposición antagónica textual no recibiera una excitación similar (por ejemplo, al ser leída con poca atención), la conexión inhibitoria podría hacer que 'EN(jungla, polo)' quedara desactivada al finalizar la fase de Integración y el lector no detectara la inconsistencia.

Un próximo experimento controlado será dedicado a la replicación del fenómeno de interacción encontrado aquí, y también a determinar cuál de las dos conjeturas anteriores es capaz de explicarlo.

Los resultados obtenidos en esta investigación deben interpretarse dentro de los límites de la misma. Estudios posteriores serán necesarios para replicar los resultados con otras de participantes y aumentar la validez externa del estudio. Además, futuras replicaciones podrían realizarse con hablantes de otras lenguas, de modo que los vínculos filológicos entre L1 y L2 sean más próximos que entre español e inglés (por ejemplo, entre catalán y español) o sean más lejanos (por ejemplo, chino e inglés, o español y alemán). 


\section{REFERENCIAS BIBLIOGRÁFICAS}

Alderson, J. C. (1984). Reading in a foreign language: A reading problem or a language problem? En J. C. Alderson \& A. H. Urquhart (Eds.), Reading in a foreign language (pp. 122-135). Nueva York: Ablex.

Bernhardt, E. B. \& Kamil, M. L. (1995). Interpreting relationships between L1 and L2 reading: Consolidating the linguistic threshold and the linguistic interdependence hypothesis. Applied Linguistics, 16(1), 15-33.

Block, E. (1986). The comprehension strategies of second language readers. TESOL Quarterly, 20(3), 463-494.

Block, E. (1992). See how they read: Comprehension monitoring of L1 and L2 readers. TESOL Quarterly, 26(2), 319-343.

Campanario, J. M. \& Otero, J. (2000). Más allá de las ideas previas como dificultades de aprendizaje: Las pautas de pensamiento. Las concepciones epistemológicas y las estrategias metacognitivas de los alumnos de ciencias. Enseñanza de las Ciencias, 18, 155-169.

Carrell, P. L. (1991). Second language reading: Reading ability or reading proficiency? Applied Linguistics, 12, 159-179.

Carrell, P. L. \& Grabe, W. (2002). Reading. En N. Schmitt (Ed.), An introduction to applied linguistics (pp. 233-250). Londres: Arnold.

Casanave, C. (1988). Comprehension monitoring in ESL reading: A neglected essential. TESOL Quarterly, 22, 283-302.

Chinn, C. A. \& Brewer, W. F. (1993). The role of anomalous data in knowledge acquisition: A theoretical framework and implications for science instruction. Review of Educational Research, 63(1), 1-49.

Clarke, M. A. (1980). The short circuit hypothesis of ESL reading or when language competence interferes with reading performance. Modern Language Journal, 64(2), 203-209.

Council of Europe (2001). Common European Framework of Reference for Languages: Learning. Teaching. Assessment. Cambridge (U.K.): Cambridge University Press.

Cummins, J. (1991). Interdependence of first-second-language proficiency in bilingual children. En E. Bialystok (Ed.), Language processing in bilingual cbildren (pp. 70-89). Cambridge: Cambridge University Press. 
Erickson, T. A. \& Mattson, M. E. (1981). From words to meaning: A semantic illusion. Journal of Verbal Learning and Verbal Behavior, 20, 540-552.

Flavell, J. H. (1981). Cognitive monitoring. En W. P. Dickson (Ed.), Children's oral communication skills (pp. 35-60). Nueva York: Academic Press.

Fukkink, R. G., Hulstijn, J. \& Simis, A. (2005). Does training in second-language word recognition skills affect reading comprehension? An experimental study. The Modern Language Journal, 89(1), 54-75.

Gómez, A., Solaz, J. J. \& Sanjosé, V. (2012). Competencia en lengua inglesa de estudiantes universitarios españoles en el contexto del EEES: Nivel de dominio lingüístico, estrategias metacognitivas y hábitos lectores. Aceptado para su publicación en Revista de Educación (DOI: 10-4438/1988-592X-RE-2012-363-175).

Grabe, W. (2009). Reading in a second language: Moving from theory to practice. Nueva York: Cambridge University Press.

Grabe, W. \& Stoller, F. L. (2002). Teaching and researching reading. Londres: Pearson.

Graesser, A. C., Hoffman, N. L. \& Clark, L. F. (1980). Structural components of reading time. Journal of Verbal Learning and Verbal Behavior, 19, 135-151.

Han, F. \& Stevenson, M. (2008). Comprehension monitoring in first and foreign language reading. University of Sydney Papers in TESOL, 3, 73-110.

Ibánez, R. (2008). Comprensión de textos académicos escritos en inglés: Relación entre nivel de logro y variables involucradas. Revista Signos. Estudios de Lingüística, 41(67), 203-229.

Kintsch, W. (1988). The role of knowledge in discourse comprehension: A construction-integration model. Psychological Review, 95(2), 163-182.

Kintsch, W. \& van Dijk, T. A. (1978). Towards a model of text comprehension and production. Psychological Review, 85, 363-394.

Koda, K. (2005). Insights into second language reading: A cross-linguistic approach. Cambridge: Cambridge University Press.

Koda, K. (2007). Reading and language learning: Crosslinguistic constrains on second language reading development. Language Learning, 57(1), 1-44.

Machida, S. (2011). Reading foreign language text-what separates skilled and unskilled FL readers? Electronic Journal of Foreign Language Teaching, 8(2), 141-153. 
Morrison, L. (2004). Comprehension monitoring in first and second language reading. The Canadian Modern Language Review, 61(1), 77-106.

Otero, J. \& Graesser, A. C. (2001). PREG: Elements of a model of question asking. Cognition and Instruction, 19, 143-175.

Otero, J. \& Kintsch, W. (1992). Failures to detect contradictions in a text: What readers believe versus what they read. Psychological Science, 3, 229-235.

Perfetti, C. A. (1985). Reading ability. Nueva York: Oxford University Press.

Segalowitz, N., Poulsen, C. \& Komoda, M. (1991). Lower level components of reading skill in higher level bilinguals: Implications for reading instruction. En J. H. Hulstijn \& J. F. Matter (Eds.), Reading in two languages (pp. 15-30). The Netherlands: AILA Review.

Taguchi, E., Gorsuch, G. J. \& Sasamoto, E. (2006). Developing second and foreign language reading fluency and its effect on comprehension: A missing link. The Reading Matrix, 6(2), 1-19.

Tsai, Y. R., Ernst, C. \& Talley, P. C. (2010). L1 and L2 strategy use in reading comprehension of Chinese EFL readers. Reading Psychology, 31(1), 1-29.

van Dijk, T. A. (1980). Macrostructures: An interdisciplinary study of global structures in discourse, interaction and cognition. Nueva Jersey: Lawrence Erlbaum.

Vosniadou, S. P., Pearson, D. P. \& Rogers, T. (1988). What causes children's failures to detect inconsistencies in text? Representation versus comparison difficulties. Journal of Educational Psychology, 80, 27-39.

Wang, M. C., Haertel, G. D. \& Walberg, H. J. (1993). Toward a knowledge base for school learning. Review of Educational Research, 63(3), 249-294.

Winograd, P. \& Johnston, P. (1982). Comprehension monitoring and the Error Detection Paradigm. Journal of Reading Behavior, 14(1), 61-76.

Yamashita, J. (2002). Reading strategies in L1 and L2: Comparison of four groups of readers with different reading ability in L1 and L2. Review of Applied Linguistics, 135-136, 1-35.

Zabrucky, K. \& Ratner, H. H. (1986). Children's comprehension monitoring and recall of inconsistent stories. Child Development, 57, 1401-1418.

\section{NOTA}

1. Las competencias asociadas a cada nivel se encuentran explicitadas, por ejemplo en: http://es.wikipedia.org/wiki/Marco_común_europeo_de_referencia_para_las_lenguas 


\section{ANEXO}

Extracto de los materiales utilizados para evaluar el control de la comprensión micro y macroestructural. Se muestran primero las instrucciones, luego el ejemplo de práctica y, finalmente uno de los textos en inglés. Las inconsistencias introducidas en cada texto se han subrayado aquí para facilitar su detección.

\section{(Por favor, no pases la página hasta que te lo indique el profesor)}

\section{ES MUY IMPORTANTE LEER CON MUCHA ATENCIÓN LAS SIGUIENTES INSTRUCCIONES}

Esta prueba forma parte de una investigación educativa sobre comprensión de textos en lengua extranjera y lengua materna. Se trata de que valores si algunos textos de ciencias son comprensibles para tus futuros estudiantes.

Muchas veces estos textos usan palabras difíciles, contienen frases confusas, incoherencias o bien presentan algún error. Nosotros investigamos la adecuación de los textos escolares a la capacidad de los estudiantes para poder controlar su propia comprensión. Para eso, utilizamos textos especialmente preparados por nosotros para este experimento. Te vamos a mostrar 3 textos en inglés y 3 en español. En ellos sería posible que encontraras palabras o frases que te parezcan inadecuadas y/o incomprensibles. En ese caso, deberás detectarlas y marcarlas como te indicamos después. La tarea consiste en:

a) LEER el texto tratando de ENTENDER completamente su contenido.

b) SUBRAYAR las palabras y/o frases que no entiendas perfectamente o te parezcan inadecuadas. Cada persona puede tener dificultades en diferentes partes de un mismo texto y por razones diferentes (diferente conocimiento de un idioma, diferentes estrategias de lectura...). No importa. Todos los criterios resultan válidos para nosotros. En los textos que hemos preparado, es posible que, por la razón que sea, encuentres palabras que te resulten difíciles de entender o frases incoherentes para ti. Pero es posible que encuentres el texto adecuado tal como está. En ese caso, no debes subrayar nada.

c) INDICAR LA RAZÓN por la que has subrayado esa(s) palabra(s) o frase(s), para que podamos saber la razón. Para esto, usarás un CÓDIGO que te daremos en cada texto.

Repetimos que no tienes por qué subrayar nada si no encuentras nada raro, erróneo, incomprensible, absurdo o incoherente en un texto. Como hemos dicho ya, encontrar problemas en un texto o no encontrarlos depende de cada persona. 
Cuando hayas acabado con un texto, deberás entregarlo antes de continuar y ya no podrás regresar a él.

Puedes pasar la página cuando el profesor lo indique

(Aquí hay un salto de página)

\section{RAZONES POR LAS QUE SE PUEDE SUBRAYAR EL TEXTO}

Subraya TODO LO QUE NO ENTIENDAS, sea por la razón que sea (excepto por razones de estilo o de gusto al escribir). Además, deberás indicar la razón concreta por la cual no se comprenden esas partes subrayadas.

Subraya SOLAMENTE LO QUE NO ENTIENDAS. En el caso de que subrayes alguna parte del texto, deberás escribir debajo del subrayado uno de estos números ' 1 ', '2' o '3', según corresponda a una de las siguientes causas:

\section{CÓDIGO}

- Escribe un '1' debajo de una parte de información que te parezca inapropiada por no ser coherente o ser contradictoria con alguna(s) otra(s) idea(s) del texto.

- Escribe un '2' debajo de una parte del texto que te resulte absurda o sin sentido.

- Escribe un ' 3 ' debajo de las palabras desconocidas para ti porque nunca la hayas visto antes, o porque no sabes tanto inglés, etc.

$\mathrm{Si}$ quieres expresar otra causa diferente a estas tres, escribe un '4' debajo del subrayado. Después, abajo, explicas qué quieres que entendamos.

\section{EJEMPLO para practicar}

La lluvia es un fenómeno meteorológico muy frecuente en gran parte de nuestro planeta. Consiste en gotas de agua que caen desde el cielo hasta la tierra. Estas gotas proceden de nubes que se han ido formando por hipertrifectación del vapor de agua. El agua existe normalmente en la atmósfera en forma de gas. Si la temperatura baja, el gas puede convertirse en líquido y se forman gotas microscópicas. A veces, las gotas se hacen demasiado secas y entonces bajan a la tierra.

En resumen, la lluvia se forma cuando el vapor de agua se convierte en pequeñas gotas debido a un calentamiento de la atmósfera. 


\title{
Explicación del profesor:
}

a) La palabra 'hipertrifectación' no la conozco. Entiendo que debería decir 'condensación', pero subrayo la palabra rara y la codifico con un ' 3 '.

b) Gotas de agua secas no existen. En este caso, 'secas' otorga un significado absurdo que debo subrayar y codificar con un ' 2 '.

c) Una idea anterior en el texto dice que el gas se convierte en líquido cuando la temperatura baja. Entonces, esta frase última es incoherente con esa idea del texto. Marco con ' 1 ' las palabras que no encajan, o toda la idea si lo prefiero.

\section{CONSERVA A LA VISTA ESTA HOJA. PUEDES CONSULTARLA TODAS LAS VECES QUE LO NECESITES}

\author{
The Arctic Sea Ice is Melting Faster
}

\section{Número de palabras: 221 Índice de dificultad lectora (Flesh-Kincaid $)=60.5$ (estándar); Nivel Europeo: A2-B1}

Greenhouse effect in the Earth is caused by heat-trapping gases like carbon dioxide in the atmosphere. An increase of the greenhouse effect will cause global warming and environmental changes. One of these changes is the reduction of the mass of sea ice floating on the Arctic Ocean.

Dr Julienne Stroeve is the author of a new study about the Arctic's ice surface. Warm waters entering the Arctic region combined with warming air temperatures are causing the destruction of the sea ice. Dr Stroeve found that since 1953 the area of hot ice in the Arctic has declined at an average rate of 7,8 per cent per decade. She compared the observed tendencies between 1953 and 2150 with the projections made by a rustic group of experts on climate change. This study estimated the ice area is decreasing at an average rate of 2,5 per cent per decade in the same period.

Summing up, sea ice on the Arctic Ocean is going up year-by-year. Climate experts may have underestimated the power of global warming from humangenerated greenhouse gases. When the concentration of carbon dioxide grows up, the greenhouse effect becomes less important. If emissions of heat-trapping gases were not significantly decreased, the Arctic region could end up with no floating ice in a few decades. 